\title{
The spectrum of male breast disease at Charlotte Maxeke Johannesburg Academic Hospital - a 3-year retrospective review
}

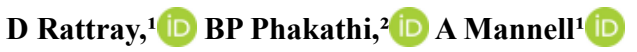 \\ ${ }^{1}$ Department of Surgery, Faculty of Health Sciences, University of the Witwatersrand, South Africa \\ ${ }^{2}$ Department of Surgery, University of the Witwatersrand and Charlotte Maxeke Johannesburg Academic Hospital, South Africa
}

Corresponding author, email: darren.rattray@gmail.com

Background: The spectrum of male breast disease (MBD) and its relative proportions is not well documented. This study aims to describe the demographics, clinical, radiological and histopathological characteristics of the spectrum of MBD managed at Charlotte Maxeke Johannesburg Academic Hospital (CMJAH).

Methods: This is a retrospective, descriptive study of all male patients diagnosed with MBD at CMJAH between 1 January 2016 and 31 December 2018. Patients' data were extracted from the Breast Imaging Department, CMJAH Breast Clinic and the National Health Laboratory Services patients' records. Data collected included patients' demographics, clinical presentation, radiological findings and histopathological diagnosis, where available. The collected data were captured using REDCap ${ }^{\mathrm{TM}}$ and were analysed using Statistica 13 and SAS version 9.2. $P$-value of 0.05 was used for statistical significance.

Results: Of the 269 males imaged, 244 (91\%) had a diagnosed breast condition, 90\% of which were benign. Gynaecomastia accounted for $85 \%$ of all breast disease diagnosed. Patients who presented with benign breast disease were significantly younger than those with malignant breast disease, with a mean age of 45.59 years vs 58.29 years $(p=0.0007)$. Seventyone per cent of patients had a known HIV status with $39 \%$ being HIV positive. There was a significant association between patients with HIV and benign breast disease $(p=0.0129)$.

Conclusion: Gynaecomastia is the most common MBD seen at CMJAH. There was a significant association between HIV and benign breast disease. This association should be explored further with respect to the direct effects of the virus and to those of the antiretroviral medication.

Keywords: male breast disease, Charlotte Maxeke Johannesburg Academic Hospital

\section{Introduction}

Although most commonly associated with females, breast disease may also manifest in males in a variety of conditions ranging from benign to malignant. ${ }^{1}$ Despite accounting for a large proportion of outpatient department presentations, due to the relative rarity of male breast disease (MBD), in conjunction with lack of awareness, it often goes under recognised. ${ }^{2}$ Some of this may have to do with the social stigma MBD carries. Gynaecomastia is the most common benign breast disease in males, ${ }^{2}$ and due to its association with human immunodeficiency virus (HIV) and anti-retroviral therapy (ART), is of particular interest in the South African context, with an HIV prevalence of $12.9(9.3-14.3)$ per 100000 men between the ages of 15 and $49 .{ }^{3}$ In a 9-year retrospective review of HIV positive patients with breast-related pathology, Pantanowitz et al. found that of the HIV positive men with breast-related pathology, $58 \%$ had gynaecomastia related to ART of all drug classes. ${ }^{4}$ A 2016 South African study found that $11 \%$ of adverse drug reactions (ADR) reported to the National HIV and Tuberculosis Health Care Worker Hotline between June 2013 and July 2014 were for gynaecomastia, ${ }^{5}$ while another found gynaecomastia to be the 4 th $(8.8 \%)$ most common
ART-associated ADR in South Africa. ${ }^{6}$ This highlights the significance a diagnosis of HIV has when considering benign diseases of the male breast. In Uganda, a 10-year retrospective review of histopathology reports found gynaecomastia (44\%), fibrocystic breast disease (22\%) and infiltrating ductal carcinoma $(17 \%)$ to be the three most common diagnoses among male patients. ${ }^{7}$ Furthermore, in a Turkish study which looked at all MBD diagnosed by triple assessment, gynaecomastia was the most common diagnosis $(87 \%)$, followed by primary breast carcinoma $(6 \%)$ and fat necrosis $(2 \%){ }^{8}$

Despite only accounting for less than $1 \%$ of all male cancers and less than $1 \%$ of all breast cancers, ${ }^{9,10}$ male breast cancer (MBC) constitutes the largest proportion of the literature pertaining to MBD. The reported incidence of MBC in sub-Saharan Africa is increasing, ${ }^{7,9,11}$ but as it is so rare the diagnosis may initially not be considered and, for this reason, a thorough understanding of all MBD, benign and malignant, is essential for timely and accurate diagnosis. ${ }^{10}$ One meta-analysis reviewing data across 27 African countries, revealed that the male-to-female ratio of breast cancer in Africa was 1:24, higher than in other parts of the world, ${ }^{9}$ such as America with an overall male-to-female 
ratio of $1: 100 .{ }^{12}$ Of interest, this ratio was even higher in sub-Saharan Africa than compared to North Africa. This was in keeping with a study by Bunkley et al. ${ }^{13}$ that reported a male-to-female breast cancer ratio of 1:14 across 7 subSaharan countries, one of which was South Africa, while a third study reported a geographical band in Africa displaying a male-to-female ratio of $1: 17 .{ }^{14}$ These researchers also found that the average age at presentation amongst men with breast cancer was 54.6, seven years older than women with breast cancer, versus 67 and 61 for men and women respectively, published in data from the United States. ${ }^{9}$ A 2017 study by Motloutsi and Lubinga ${ }^{15}$ suggested that there may be a genetic explanation for the prevalence of breast cancer in African men. This is supported by the findings of an increased incidence of MBC in sub-Saharan Africa and a 2017 report from the Cancer Association of South Africa, which states that more than $50 \%$ of MBC patients were African men. ${ }^{16}$

This study aims to describe the spectrum of MBD at Charlotte Maxeke Johannesburg Academic Hospital (CMJAH), South Africa, reviewing the demographics, clinical, radiological and, where available, the histopathological characteristics of MBD at this single institution.

\section{Methods}

\section{Study design}

This was a retrospective, descriptive study of all male patients who presented at CMJAH with breast disease between 1 January 2016 and 31 December 2018. File numbers of all male patients who underwent breast imaging during the review period were obtained from the CMJAH Breast Imaging Department. Using the patients' file numbers, data relevant to the study were collected from various databases including the CMJAH picture archiving and communication system (PACS), the National Health Laboratory Service (NHLS) database, and patient medical records from the CMJAH Breast Clinic. Data were then entered into a preformulated data collection sheet using REDCap ${ }^{\mathrm{TM}}$, in a double password protected (computer password and database password) online database. Each patient received a study number, providing the only link to the patient's details. Data collected included patient's age at presentation, self-identified ethnicity, HIV status, presenting complaint, radiological modality, radiological findings as a specific diagnosis and/or BI-RADS grading, and histopathological diagnosis where available. HIV status was established looking at documentation on radiological reports, a search of the NHLS database and/or review of patients' records. Data were then extracted from REDCap ${ }^{\mathrm{TM}}$ in the form of an Excel spreadsheet and analysed using Statistica 13 and SAS version 9.2. Descriptive statistics, (frequencies and percentages), were calculated for categorical data, and means and standard deviations were calculated for numerical data. Analytical statistics, the independent t-test and analysis of variance (ANOVA), were used to compare the mean values in two and three groups respectively. Fisher's exact test was used to compare percentages in different groups. The $p$-value of 0.05 was used for statistical significance.

\section{Results}

\section{Demographics}

During the study period a total of 274 males underwent breast imaging. There were no breast imaging reports available for five men, leaving 269 males included in the study. In total, $94 \%(n=252)$ of these men were first presentations, while the remaining $6 \%(n=17)$ were follow-up patients. Of the men who presented for the first time, the mean age was 46 $(\mathrm{SD} \pm 17.6)$ years, the median age 45 , and a range of 13 to 84 years (Figure 1). In terms of ethnicity, $230(86 \%)$ of the men imaged were black, $30(11 \%)$ were white, five $(2 \%)$ were coloured and four (1\%) were Indian. Thirty-nine per cent $(n=105)$ were documented to be HIV positive, $32 \%$ $(n=86)$ HIV negative, and $29 \%(n=78)$ were HIV status unknown (Table I).

\section{Breast assessment}

The most common presenting complaint was breast enlargement $(n=152,57 \%)$, followed by a mass $(n=96,36 \%)$ and mastalgia $(n=30,11 \%)$. Of the 269 men imaged, 254 (94\%) had mammographic imaging as well as ultrasound, while the remaining $15(6 \%)$ only had an ultrasound. Of the breast imaging reports, $75 \%(n=201)$ gave both a specific diagnosis and a BI-RADS grading, 19\% $(n=51)$ gave only a BI-RADS grading, and $6 \%(n=17)$ only a specific diagnosis. Thirty-seven males had histologically confirmed

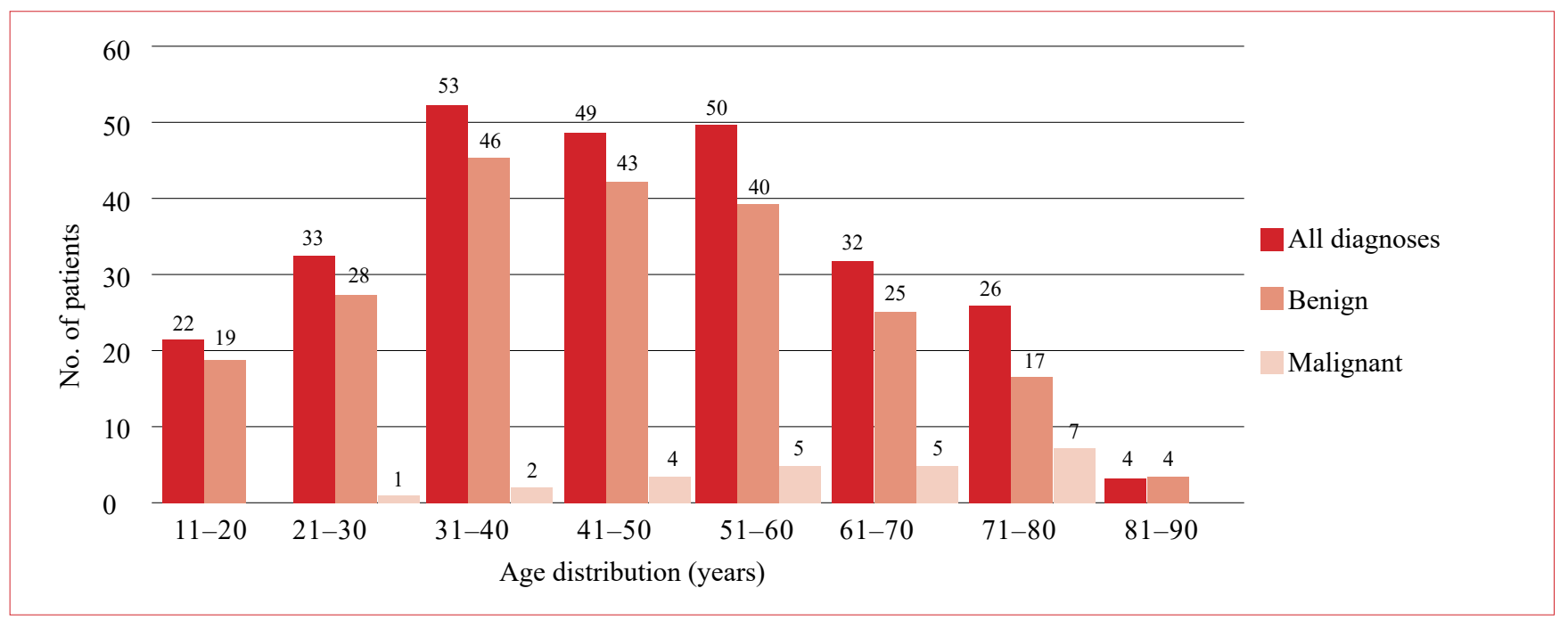

Figure 1: Age distribution of men at presentation for breast imaging 


\begin{tabular}{lc} 
Table I: Patient characteristics & \\
\hline Total number of patients & 269 \\
First presentations & $252(94 \%)$ \\
Follow-ups & $17(6 \%)$ \\
Age at first presentation & $46 \pm 17.6$ \\
Race & \\
\hline Black & $230(86 \%)$ \\
White & $30(11 \%)$ \\
Coloured & $5(2 \%)$ \\
Indian & $4(1 \%)$ \\
HIV status & \\
\hline Positive & $105(39 \%)$ \\
Negative & $86(32 \%)$ \\
Unknown & $78(29 \%)$ \\
Presenting complaint & \\
\hline Enlargement & $152(57 \%)$ \\
Mass & $96(36 \%)$ \\
Mastalgia & $30(11 \%)$ \\
Skin changes & $1(<1 \%)$ \\
Nipple discharge & $4(1 \%)$ \\
Follow-up & $17(6 \%)$ \\
Other & $6(2 \%)$ \\
\hline &
\end{tabular}

breast disease. Invasive carcinoma $(n=16)$ was the most common histopathologically diagnosed MBD, followed by gynaecomastia $(n=10)$. Other notable diseases diagnosed histopathologically included tuberculosis $(n=1)$ and dermatofibrosarcoma protuberans (DFSP) $(n=1)$. Of the 269 men who underwent breast imaging, 244 (91\%) had a diagnosed breast condition. Two hundred and twenty
(90\%) of the diagnoses were benign, and $24(10 \%)$ were malignant, with or without a concomitant benign diagnosis. Of the patients with malignant disease, seventeen were newly diagnosed, six were known with malignant disease, and one was a recurrence. The most common breast disease diagnosed was gynaecomastia, accounting for $85 \%$, while breast cancer accounted for $10 \%$ of cases (Figure 2).

\section{Associations}

Patients' age was significantly associated with benign vs malignant breast disease, with mean ages of 45.59 years and 58.29 years respectively $(p=0.0007)$. There were no significant associations between ethnicity and benign vs malignant breast disease $(p=1.0000)$, or disease subcategories (categorised as gynaecomastia, carcinoma or other) ( $p=0.3985)$. The greatest proportion of benign breast disease occurred in patients who were HIV positive, while most of malignant breast disease occurred in patients who were HIV negative $(p=0.0129)$. Regarding disease subcategories, a significantly greater number of patients with gynaecomastia were HIV positive $(p=0.0111)$.

\section{Discussion}

\section{Spectrum of male breast disease}

The main aim of this study was to identify the spectrum of MBD presenting at CMJAH over a 3-year period. There are very few studies which looked specifically at the spectrum of breast disease amongst males, and only one which shared a similar demographic profile to this study. There were only two that could be used to make a comparison.

This study, which included all MBDs diagnosed radiologically and/or histopathologically, identified a total of 269 males, diagnosed with 16 different MBDs over a period of three years. A study from Uganda, which only looked at

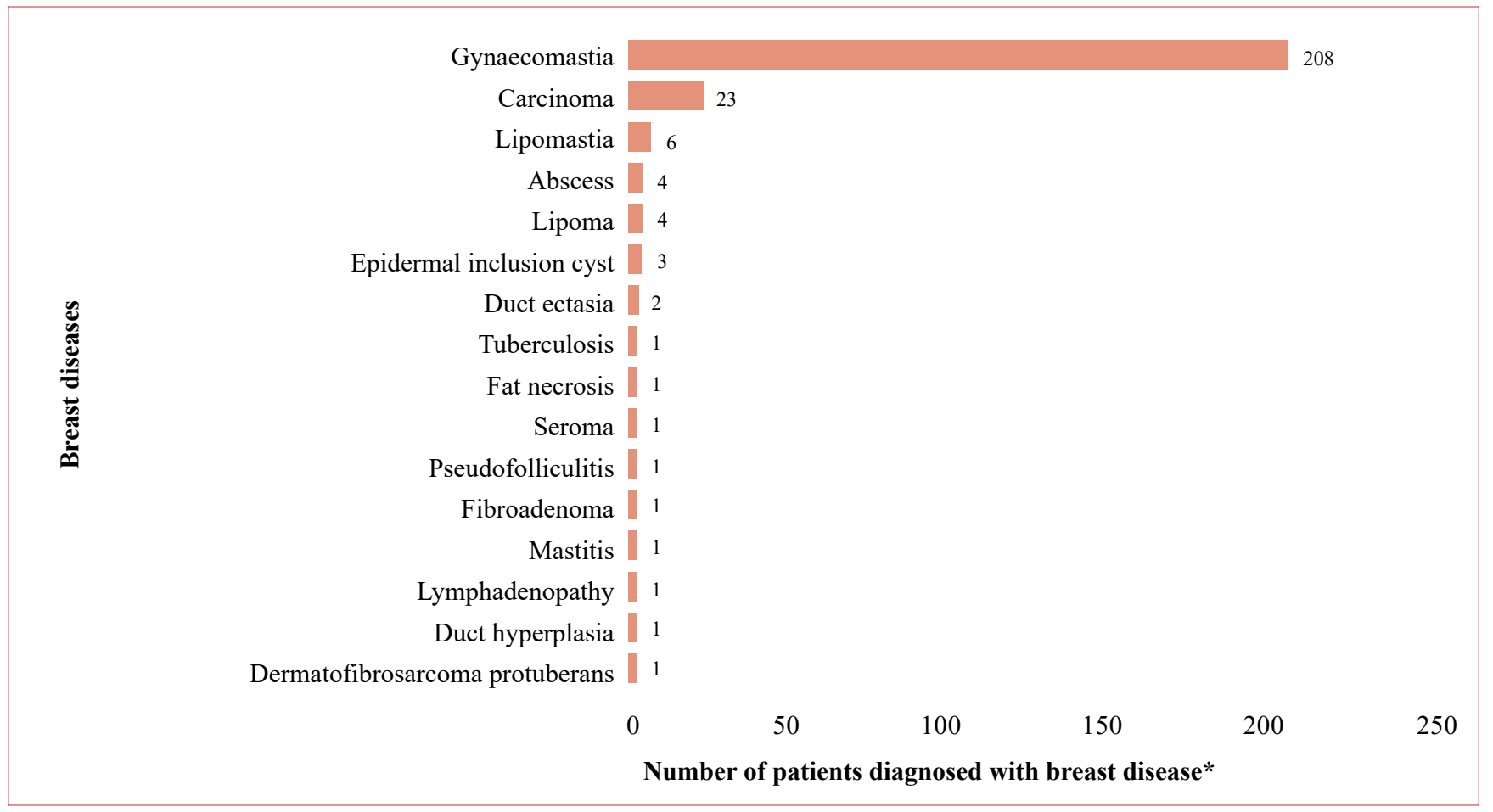

Figure 2: Spectrum of male breast disease identified during the study period

* Although 244 men were diagnosed with breast disease, 15 men had concomitant diagnoses, bringing the total number of diagnosed breast diseases to 259 
histopathologically diagnosed MBD, identified a total of 51 males, diagnosed with eight different MBDs over a period of ten years. Gynaecomastia was the most common diagnosis, followed by fibrocystic breast disease and infiltrating ductal carcinoma. ${ }^{7}$ By comparison, the current study identified a total of 37 males with histopathologically confirmed MBD, diagnosed with nine different pathologies, over a period of three years: invasive carcinoma was the most common histopathological diagnosis, followed by gynaecomastia. This suggests that CMJAH may diagnose more men with MBD histopathologically and see a wider variety of pathology than identified in Uganda. The most common diagnoses were proportionately different in each study. While the CMJAH Breast Imaging Department follows a strict protocol regarding which patients warrant biopsies based on their BI-RADS grading, it is not evident if the same protocol was followed in the study done by Ssemmanda et al. ${ }^{7}$ This could explain the difference between the most common diagnoses identified by each study. In the Ugandan study it is possible that a greater number of males underwent biopsies for conditions which would be diagnosed radiologically at the CMJAH Breast Imaging Unit.

As in the study by Günhan-Bilgen et al., ${ }^{8}$ the current study also found gynaecomastia ( $87 \%$ vs $85 \%$ ) and primary breast carcinoma ( $6 \%$ vs $9 \%$ ) to be the most commonly diagnosed breast diseases amongst males. Other conditions such as fat necrosis, lipoma, abscess and epidermal inclusion cysts were also found to be present in similar proportions.

\section{Male breast cancer}

This study identified 24 males with male breast cancer. Six patients were known with MBC, 17 patients were newly diagnosed with a breast malignancy during the study period, and one was a recurrence. Fifteen were invasive ductal carcinomas, one case of DFSP and in one case no consensus could be made regarding malignant sub-type. The average age at presentation of those newly diagnosed with $\mathrm{MBC}$ was 56.8 years. This is in keeping with a 2012 meta-analysis of all MBC in Africa by Ndom et al. who found that the average age at presentation amongst men with breast cancer was 54.6.9

\section{Male breast disease and HIV}

Gynaecomastia is a well-recognised complication of HIV and ART use ${ }^{4-6}$ and, as was suggested in other studies, the current study also identified a significant association between HIV positive males and benign breast disease, specifically gynaecomastia. While Pantanowitz et al. ${ }^{4}$ found this association to be due to ART of all drug classes, as this study did not record ART use or regimen, the same association could not be made. HIV is also associated with infectious breast diseases such as tuberculous mastitis and pyogenic abscess. ${ }^{4}$ Of the five patients identified with breast abscesses or mastitis in this study, only two (40\%) were HIV positive, and another two were HIV status unknown. TB mastitis, once considered a rarity, now accounts for $3-4 \%$ of all breast diseases in regions with a high incidence of TB, such as Africa. ${ }^{14}$ However, TB infection of the male breast is scarcely documented. Interestingly, the only patient documented with tuberculous mastitis in the current series was found to be HIV negative. This suggests that more aggressive screening for HIV should be done in males presenting with infectious breast conditions.

\section{Study limitations}

The retrospective nature of this study lends it to some degree of selection bias. As there were no reports available for five of the 274 males who underwent breast imaging during the study period, the missing reports may have resulted in skewing of results. A second limitation of this study was the failure to record clinical examination findings, a major component of the triple breast assessment. Another limitation was the failure to record the details of ART use in the HIV positive patients, something not routinely recorded in patient records. This means that, while there is an association between HIV and gynaecomastia, this study could not confirm whether the association is due to ART or HIV alone. This could be addressed in a future prospective study. Lastly, as not all patients were biopsied, the radiological diagnosis assigned to patients could not be absolutely confirmed.

\section{Conclusion}

Gynaecomastia is the most common MBD seen at CMJAH, and in the literature reviewed. There was a significant association between HIV positive status and benign breast disease, an area which deserves to be explored further with regard to the direct (viral) and indirect (treatment) effects of HIV on male breast diseases.

\section{Conflict of interest}

The authors declare no conflict of interest.

\section{Funding source}

None.

\section{Ethical approval}

This study was approved by the University of Witwatersrand Human Research Ethics Committee, clearance certificate number M191127.

\section{ORCID}

D Rattray (i) https://orcid.org/0000-0002-1597-3097

BP Phakathi (D) https://orcid.org/0000-0002-8991-6060

A Mannell (iD https://orcid.org/0000-0003-4438-4045

\section{REFERENCES}

1. Chesebro AL, Rives AF, Shaffer K. Male breast disease: what the radiologist needs to know. Curr Probl Diagn Radiol. 2019;48(5):482-93

2. Yen PPW, Sinha N, Barnes PJ, Butt R, Iles S. Benign and malignant male breast diseases: radiologic and pathologic correlation. Can Assoc Radiol J. 2015 Aug;66(3):198-207.

3. Country Factsheets. South Africa, 2019 [Internet]. www. unaids.org. Available from: https://www.unaids.org/en/ regionscountries/countries/southafrica. Accessed 17 Aug 2020.

4. Pantanowitz L, Sen S, Crisi GM, et al. Spectrum of breast disease encountered in HIV-positive patients at a community teaching hospital. Breast. 2011 Aug;20(4):303-8

5. Njuguna C, SwartA, Blockman $M$, et al. Cases of antiretroviralassociated gynaecomastia reported to the National HIV \& Tuberculosis Health Care Worker Hotline in South Africa. AIDS Res Ther. 2016;13:40. 
6. Birbal S, Dheda M, Ojewole E, Oosthuizen F. Adverse drug reactions associated with antiretroviral therapy in South Africa. African J AIDS Res. 2016 Sep;15(3):243-8.

7. Ssemmanda S, Katagirya E, Bukirwa P, et al. Breast diseases histologically diagnosed at a tertiary facility in Uganda (20052014). BMC Cancer. 2018 Dec 22;18(1):1285.

8. Günhan-Bilgen I, Bozkaya H, Üstün EE, Memiş A. Male breast disease: clinical, mammographic, and ultrasonographic features. Eur J Radiol. 2002 Sep;43(3):246-55.

9. Ndom P, Um G, Bell EMD, et al. A meta-analysis of male breast cancer in Africa. Breast. 2012 Jun;21(3):237-41.

10. Chau A, Jafarian N, Rosa M. Male breast: clinical and imaging evaluations of benign and malignant entities with histologic correlation. Am J Med. 2016 Aug;129(8):776-91.

11. Hoda SA, Brogi E, Koerner FC, Rosen PP. Rosen's Breast Pathology: 4th ed. Philadelphia: Lippincott Williams \& Wilkins; 2014.
12. Korde LA, Zujewski JA, Kamin L, et al. Multidisciplinary meeting on male breast cancer: summary and research recommendations. J Clin Oncol. 2010 Apr 20;28(12):2114-22.

13. Bunkley DT, Robinson JD, Bennett NE, Gordon S. Breast cancer in men: Emasculation by association? J Clin Psychol Med Settings. 2000;23(3):653-62.

14. Marinopoulos S, Lourantou D, Gatzionis T, et al. Breast tuberculosis: diagnosis, management and treatment. Int J Surg Case Rep. 2012;3(11):548-50.

15. Motloutsi A, Lubinga E. Communicating male breast cancer: knowledge and awareness among some South African youth. Communitas. 2017;22:87-97. https://doi. org/10.18820/24150525/Comm.v22.7.

16. Herbst M. Fact Sheet and Position Statement on Soursop (Graviola). Cancer Association of South Africa (CANSA). 1 Jul 2013. 